\title{
Correction: Autoantibodies to synapsin I sequestrate synapsin I and alter synaptic function
}

\author{
Anna Rocchi, Silvio Sacchetti, Antonio De Fusco, Silvia Giovedi, Barbara Parisi, Fabrizia Cesca, Markus Höltje, \\ Klemens Ruprecht, Gudrun Ahnert-Hilger and Fabio Benfenati
}

\section{Correction to: Cell Death and Disease \\ https://doi.org/10.1038/s41419-019-2106-z \\ published online 14 November 2019}

Since online publication of this article, the authors noticed that in the legend of Fig. 5, the legend to "panel e" was mistakenly omitted. The full text should read as follows:
Fig. 5 a Representative images of SynI-mAb $(1.5 \mu \mathrm{g} / \mathrm{mL})$ treated hippocampal neurons incubated either with sodium azide $\left(\mathrm{NaN}_{3} ; 500 \mathrm{nM}\right)$, PitStop $2(30 \mu \mathrm{M})$ or $\alpha$ $\mathrm{Fc} \gamma \mathrm{R}$ II/III antibody at the indicated concentrations for $72 \mathrm{~h}$. The control condition indicates cells exposed to SynI-mAb in the presence of the $\mathrm{NaN}_{3}$ /PitStop2 solvent. After treatments, cells were fixed, permeabilized and incubated with anti-mouse fluorescent secondary antibodies (green). Double staining for $\beta$ III-tubulin (blue) was performed after fixation. Scale bars, $10 \mu \mathrm{m}$. b Quantification of the immunoreactivity intensity of internalized SynI-mAb. Graph shows mean \pm SEM, single neuron data points independent preparations; ${ }^{* *} p<0.01,{ }^{* * * *} p<0.001$, one-way ANOVA/Dunnett's tests versus control. c Mean frequency and amplitude of mEPSCs (upper panels) and mIPSCs (lower panels) in hippocampal neurons treated with either SynI-mAb $(1.5 \mu \mathrm{g} / \mathrm{mL})$ or vehicle for $72 \mathrm{~h}$ in the presence or absence of $500 \mathrm{nM} \mathrm{NaN}$. d Mean frequency and amplitude of mEPSCs (upper panels) and mIPSCs (lower panels) in hippocampal neurons treated with either control CSF or patient \#1 CSF $(1.5 \mu \mathrm{g} / \mathrm{mL})$ for $72 \mathrm{~h}$ in the presence or absence of $500 \mathrm{nM} \mathrm{NaN}_{3}$. Graphs show means \pm SEM, single neuron data points from three independent preparations; " $p<0.05,{ }^{* *} p<0.01$, two-way ANOVA/Bonferroni's tests. e Expression of FcyII/III receptors in WT and SynI KO hippocampal neurons. Representative images of primary WT and SynI KO hippocampal neurons at 14 .

This has been corrected in the PDF and HTML versions of the article.

The authors apologise for any inconvenience caused. 